\title{
Antisense-Technologien in der Dermatologie: von der E. Dippel Signaltransduktion zur klinischen Anwendung
}

\author{
Antisense Technology in Dermatology: From Signal Transduction \\ to Clinical Application
}

\section{Zusammenfassung}

Die Antisense-Technologie nutzt Oligonukleotide, die mit RNAZielstrukturen über Watson-Crick-Bindungen hybridisieren und dann nachfolgend zur Degradation der Ziel-RNA führen. Antisense-Oligonukleotide wurden bereits erfolgreich angewandt, um Signaltransduktionsprozesse zu untersuchen und weiterentwickelt zur therapeutischen Anwendung bei einer Reihe von Erkrankungen, insbesondere bei Krebserkrankungen. Dabei wurden viele grundlegende Erfahrungen über die Chemie, die pharmakologischen, pharmakinetischen und toxikologischen Eigenschaften der Antisense-Oligonukleotide gesammelt. Besonders wertvoll war der Einsatz von Antisense-Oligonukleotiden bei der Untersuchung von spezifischen Signaltransduktionsabläufen der humanen Zelle. Zusammenfassend werden hier Entwicklungsprozesse skizziert und exemplarisch dargestellt.

\section{Abstract}

Antisense technology exploits oligonucleotide analogs to bind to target RNAs via Watson-Crick by hybridization and induces the degradation of the target RNA. The antisense oligonukleotide has been used successfully to investigate signal transduction processes and has been further developed as a therapeutic tool in many diseases, especially in cancer. A great deal has been learned about the basic mechanisms of antisense, the medicinal chemistry, the pharmacological, pharmacokinetic and toxicological properties of antisense molecules. Antisense technology has proven of great value in analysing the specificity of signaltransduction pathways. In this paper, the progress is summarized and exemplary the usefullness of the technology is discussed.
Es existieren eine ganze Reihe von Möglichkeiten, zelluläre Prozesse und insbesondere die intrazelluläre Signaltransduktion zu beeinflussen. Eine attraktive Alternative ist die Antisense-Technologie. Es handelt sich dabei um Einzellstrang-DNS-Sequenzen, die komplementär mit den entsprechenden Basensequenzen der Zielproteine nach dem Prinzipien der Watson-und-Crick-Basenpaarung hybridisieren. Der daraus resultierende Komplex aktiviert das Enzym RNase H, das die spezifische mRNA zerstört und die Proteinsynthese verhindert. Dieses Prinzip der Steuerung der Proteinexpression ist in den letzten 10 Jahren intensiv auf zellulärer Ebene erforscht, und bei einigen dermatologischen Erkrankungen zur klinischen Anwendung weiterentwickelt worden.

\section{Das Prinzip der Antisense-Anwendung}

Antisense-Oligonukleotide (ASO) sind kurze DNS-Sequenzen, die aus typischerweise 16-24 Basen bestehen und komplementär zur entsprechenden mRNA-Sequenz des Zielproteins ausgewählt wurden. Die Bindung der ASO and die komplementäre mRNA erfolgt nach den Prinzipien der Watson-und-Crick-Basenpaarung. Der Komplex aus ASO und mRNA aktiviert das Enzym RNase $\mathrm{H}$, welches dann die mRNA zerschneidet. Wird die Anzahl der spezifischen mRNA intrazellulär deutlich reduziert, resultiert daraus auch eine verminderte Produktion des Targetproteins. Aber auch die ASO werden durch intra- und extrazelluläre Enzyme zerstört. 
Um die Halbwertszeit der ASOs zu verlängern und damit ihre Effektivität zu erhöhen, werden ASOs chemisch modifiziert. Die bekannteste Modifikation sind so genannte Phosphodiester Backbones, d.h. die Bindung zwischen den Basen wurde durch den Austausch eines Sauerstoffatoms durch ein Schwefelatom modifiziert. Phosphotioat-ASOs besitzen durch ihre erhöhte Resistenz gegenüber endogenen Nukleasen eine längere Halbwertszeit, ohne dass die Hybridisierung mit den spezifischen Target-RNAs wesentlich verändert ist. Die Modifikation ist jedoch auch für eine erhöhte Toxizität verantwortlich bzw. in der klinischen Anwendung für reversible Nebenwirkungen wie Hypotension, Verlängerung der partiellen Tromboplastin-Zeit und Bradykardie.

Antisense-Anwendung zur Identifikation von Prozessen der Signaltransduktion

Die Anwendung von ASOs auf zellulärer Ebene kann unterschiedlich erfolgen. Ein sicheres Verfahren ist die intranukleäre Injektion von ASOs. Dabei werden mit Hilfe eines Mikromanipulators die ASO appliziert. Ausgangspunkt der Untersuchung war die Identifikation von G-Proteinen, welche für die Signaltransduktion von Adenosin-3-Rezeptoren $\mathrm{A}_{3}$ zur Aktivierung der Phospholipase $C$ verantwortlich sind. Die Adenosinrezeptoren umfassen eine Gruppe von 4 heptahelikalen Rezeptoren, wie $\operatorname{der} A_{1^{-}}, A_{2 A^{-}}, A_{2 B^{-}}$und $A_{3}$-Rezeptor. [1]. G-Proteine koppeln funktionell mit heptahelikalen Rezeptoren, um mit Effektorproteinen (Adenylyl Cyclasen, Phospholipase C- $\beta$ und Ionenkannälen) zu interagieren. Die G-Proteine bestehen aus 3 Untereinheiten $\alpha, \beta$ und $\gamma$, von denen $23 \alpha, 5 \beta$, und $11 \gamma$ bisher identifiziert wurden [2-8]. Diese werden in 4 Unterfamilien eingeteilt Gs, Gi, Gq und G12, aufgrund ihrer Homologie zur $\alpha$-Untereinheit. Die Bindung eines Liganden an den Rezeptor führt zu einer Konformationsänderung des Rezeptors, die einen Austausch von GDP zu GTP an der G-Protein $\alpha$-Untereinheit bewirkt und zu einer Dissoziation von $\mathrm{G} \alpha$-GTP und dem $\beta \gamma$-Dimer führt. Beide, G $\alpha$ und freies G $\beta \gamma$, können Effektorproteine aktivieren [9].

Zur Untersuchung diente die Rattenmastzelllinie RBL-2H3-hm1, die den $\mathrm{A}_{3}$-Rezeptor exprimiert und den stabil transfizierten muscarinischen Rezeptor $\mathrm{m} 1$ besitzt [10]. Der $A_{3}$-Rezeptor kann über Adenosin oder, wie hier gezeigt, mit dem Adenosin Analogon NECA (5-n-ethylcarboxamidoadenosine) aktiviert werden. In der nachfolgenden Signaltransduktionskaskade wird die PLC- $\beta$ aktiviert, die zur Erhöhung von Inositolphosphaten und dann zur intrazellulären $\mathrm{Ca}^{++}$-Erhöhung führt.

Bei dieser Zelllinie führte die Stimulation sowohl mit NECA als auch mit Carbachol, einen m1-Antagonisten, zur intrazellulären $\mathrm{Ca}^{++}$-Erhöhung. Die Messung der Einzelzellen erfolgte fluorometrisch. Wurden die Zellen mit Pertussistoxin vorbehandelt, war keine $\mathrm{Ca}^{++}$nach Stimulation mit NECA nachweisbar, jedoch nach Stimulation mit Carbachol. Bei Carbachol konnten wir in zurückliegenden Arbeiten zeigen, dass der m1-Rezeptor über ein Pertussis-insensitives Gq/G11-Protein die PLC- $\beta$ aktiviert [11]. Nach Stimulation des $\mathrm{A}_{3}$-Rezeptors mit NECA musste man also von einem Rezeptor-gekoppelten-Gi-Protein ausgehen. Zur genauen Identifikation der bekannten Gi-Proteine Gi1, Gi2 oder Gi3 wurden Antisense-Oligonukleotide intranukleär mit zwei unter- schiedlichen Methoden appliziert. Eine Methode bestand in der o.g. intranukleären Injektion von Antisense-Oligonukleotiden

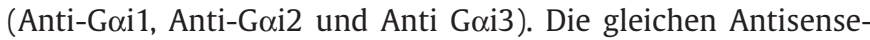
Oligonukleotide wurden mit ballistischem Transfer auf Goldpartikel appliziert. Die letztgenannte Methode soll hier kurz vorgestellt werden.

\section{Ballistischer Transfer von Antisense-Oligonukleotiden}

Für den ballistischen Transfer werden $1-10 \times 10^{6}$ Zellen auf Petrischalen für $1 \mathrm{~h}$ kultiviert. Vor der ballistischen Applikation wurden Goldpartikel zusammen mit magnetischen Beads und Antisense-Oligonukleotiden inkubiert. Der ballistische Transfer der Partikel erfolgte mit PDS-1000/He (BioRad). Getroffene Zellen wurden mit Hilfe der magnetischen Beads aussortiert und für weitere $72 \mathrm{~h}$ kultiviert. Diese Zellen wurden dann in einem weiteren Versuch mit Agonisten wie NECA oder zur Kontrolle Carbachol stimuliert und die $\mathrm{Ca}^{++}$-Mobilisation wurde mit Hilfe des Furochroms Fura-2 mit einem Einzell-Imaging-System gemessen.

Dabei zeigte sich, dass bei Zellen, die mit Anti-Goi3-AntisenseOligonukleotiden behandelt wurden, keine $\mathrm{Ca}^{++}$-Mobilisation nach Stimulation mit NECA nachweisbar war. Mit einer nachfolgenden zweiten Stimulation mit Carbachol (m1-Agonist) konnte jedoch eine $\mathrm{Ca}^{++}$-Mobilisation ausgelöst werden (Abb.1a). Zur weiteren Kontrolle der Experimente wurde auch die nichtmagnetische Fraktion mituntersucht, die jeweils das gleiche Reaktionsmuster zeigte (Abb.1 b).

Immunhistologische Untersuchungen zeigten auch, dass das Goi3-Protein durch die Antisense-Behandlung weitgehend unterdrückt wurde (Abb.2). Mit diesen Untersuchungen konnte das G-Protein identifiziert werden, welches mit dem Adenosinrezeptor $\mathrm{A}_{3}$ koppelt, um nachfolgend die Phospholipase- $\beta$ bzw. die $\mathrm{Ca}^{++}$-Mobilisation zu aktivieren.

\section{Anwendung von Antisense-Molekuilen bei Hauterkrankungen}

Die lokale Anwendung von Antisense-Molekülen an der Haut im Bereich erkrankter Hautläsionen ist eine nahe liegende und attraktive Idee. Hier sind jedoch viele Fragen im Vorfeld zu beantworten. Zunächst ist es wichtig, die Aufnahme und Internalisierung von Antisense-Oligonukleotiden sicherzustellen und zu überprüfen. Dabei können zum Teil Techniken mit fluoreszierenden Antisense-Molekülen gewählt werden, die eine Gewebelokalisation invivo zulassen. Die Aufnahme von Antisense-Nukleotiden ist von vielen Variablen abhängig. Die Wichtigsten sind die Größe der Antisense-Nukleotide, die chemische Zusammensetzung, chemische Modifikationen und Applikationsart (ballistischer Transfer, liposomaler Transfer, Injektion, bakterieller Transfer, viraler Transfer ...). Daneben muss die Pharmakokinetik von Antisense-Oligonukleotiden in Betracht gezogen werden und mögliche Nebenwirkungen durch z.B. chemische Modifikationen, die mit meist toxischen Reaktionen sich bemerkbar machen. Unabhängig von den technischen Schwierigkeiten Antisense-Moleküle an den Wirkort zu bringen, ist eine klare konzeptio- 


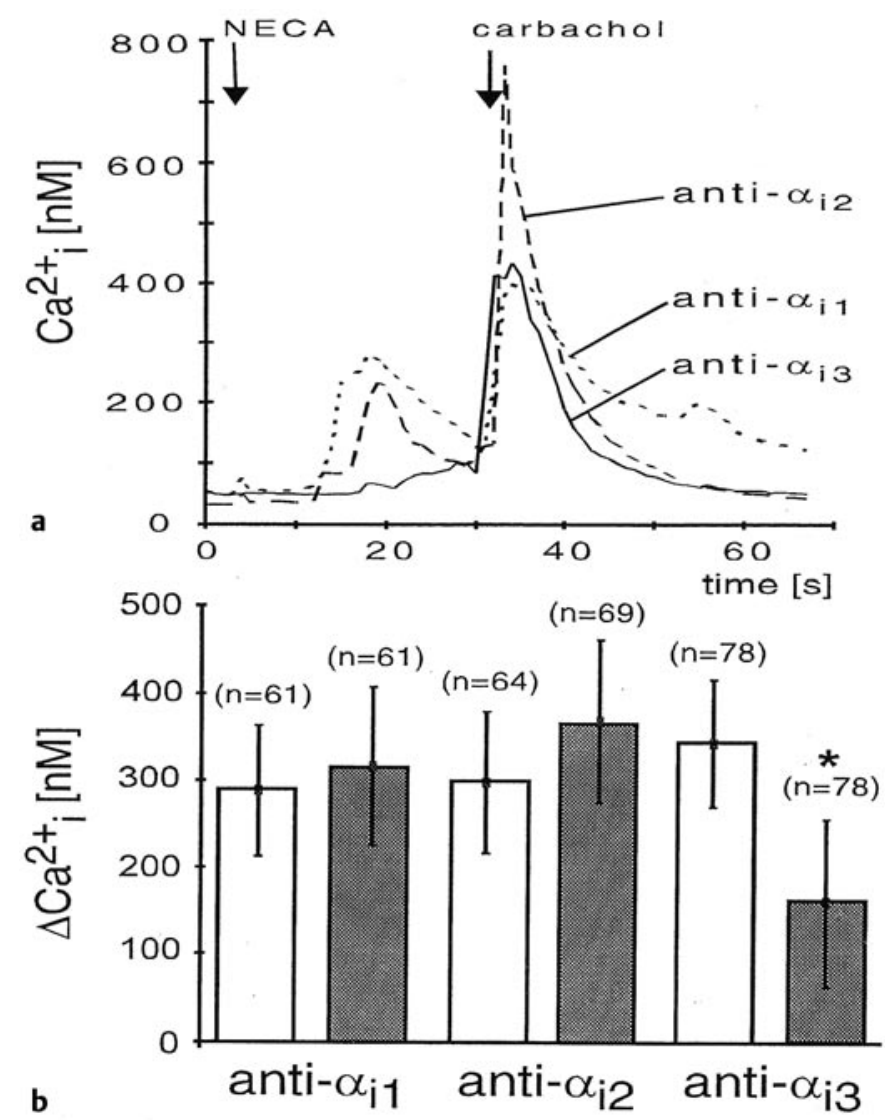

Abb. 1 a NECA-induzierter Anstieg von $\left[\mathrm{Ca}^{++}\right]_{i}$ in RBL-2H3-hm1-Zellen nach Transfektion mit Anti-Goi1, Anti-Goi2 und Anti-Goi3 ASO durch ballistischen Transfer. Jede Kurve bezieht sich auf einen Mittelwert von 15-20 untersuchten Einzelzellen. b Das Balkendiagramm zeigt die Mittelwerte des NECA-induzierten $\left[\mathrm{Ca}^{++}\right]_{i}$. Geschlossene Balken = magnetische Zellfraktion; offene Balken = nicht-magnetische Zellfraktion.

nelle Strategie wichtig, gezielt Proteine auszuschalten, die direkt mit dem Krankheitsprozess in Zusammenhang stehen, ohne die physiologischen zellulären Prozesse zu beeinflussen [12].

\section{Antisense-Moleküle in der klinischen Anwendung - Erfahrungen mit dem bcl-2-Antisense-Oligonukleotid}

Die Erfolge in der experimentellen Medizin Antisense-Moleküle gegen viele Gene einzusetzen, welche Proliferation, Angiogenese und Apoptose beeinflussen, bereitete den Weg für den Einsatz dieser Moleküle in der klinischen Anwendung und insbesondere in der Onkologie. Das mitochondriale Protein bcl-2 spielt eine zentrale Rolle bei Apoptose-Prozessen und bei vielen Tumoren. Die Überexpression dieses Proteins stellt einen wichtigen antiapoptotischen Faktor dar, der sicherlich einen wichtigen Teilaspekt für die Resistenz von Tumorzellen gegen die traditionelle zytotoxische Chemotherapie darstellt. Ein Beispiel für AntisenseOligonukleotide, welche ihren Eingang in die klinische Anwendung vollzogen haben, ist Oblimersen (Genasense ${ }^{\mathrm{TM}}$, AventisPharma/Genta Inc), ein 18-mer Antisense-Oligonukleotid, welches spezifisch humanes bcl-2-mRNA bindet [13]. Die Applikation dieses Antisense-Oligonukleotids führt zur Aktivierung des Enzyms RNAse $\mathrm{H}$ und damit zur selektiven Reduktion des anti- $\alpha_{\mathrm{i} 3}$ antibody
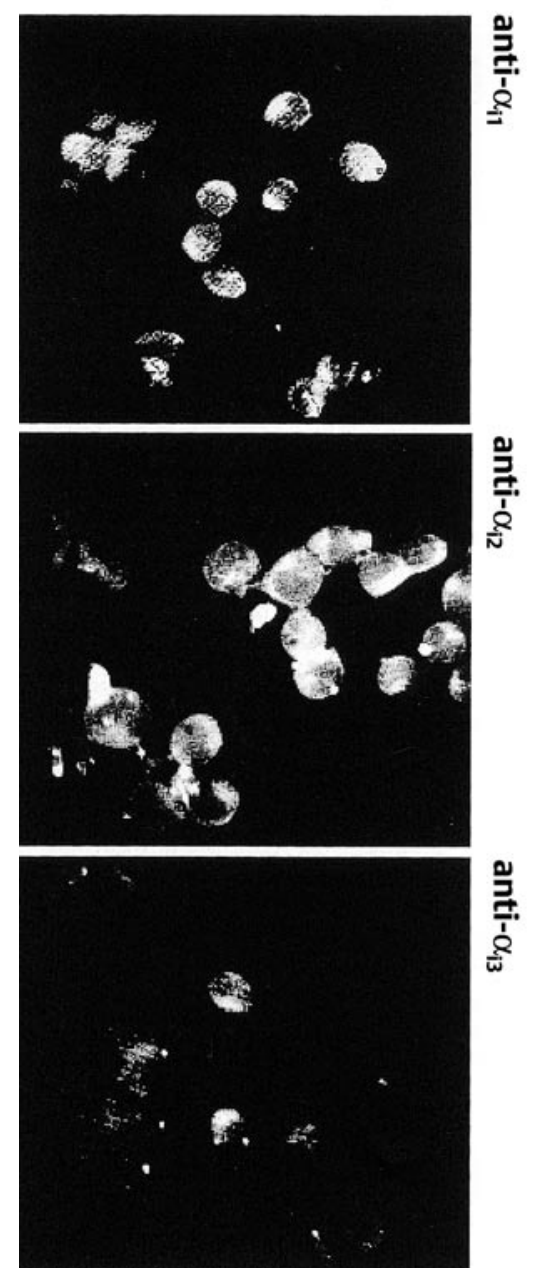

Abb. 2 Immunfluoreszenz-Untersuchung mit Antikörpern gegen das G-Protein Goi3 bei Zellen, die mit AntiGoi1, Anti-Goi2 und Anti-Goi3-ASO transfiziert wurden.
bcl-2-Proteins im Verlauf der Translation. Erfolgreiche präklinische und klinische Untersuchungen mit diesem AntisenseOligonukleotid bei unterschiedlichen Neoplasien führten zur klinischen Anwendung auch im Bereich des malignen Melanoms. Gerade beim malignen Melanom ist die Expression des Protoonkogens bcl-2 eng mit der Chemoresistenz maligner Melanomzellen verbunden. Dies konnte mit Hilfe von In-vitro-Modellen und in Maus-Modellen reproduziert werden, da nach Applikation von Antisense-Oligonukleotiden gegen bcl-2 das Protein deutlich verringert wurde und eine nachfolgende systemische Therapie mit Dacarbacin z. B. im Maus-Modell zu einer erhöhten Tumorreduktion führte. In einer klinischen Phase I - II-Studie konnte Jansen und Mitarbeiter (2000) [14] zeigen, dass multiorganmetastasierte Patienten mit malignem Melanom die Kombination aus Antisense-Molekülen und Gabe von Dacarbacin ohne eine nachweisbare dosislimitierende Toxizität gut tolerieren. Die hämatologischen Nebenwirkungen waren mild bis moderat. Höhere Dosen des Antisense-Moleküls führten zu einem kurzen transienten Fieber. Vier Patienten zeigten eine transiente Leberfunktionsstörung, welche sich innerhalb einer Woche zurückbildete. Sechs der vierzehn Patienten zeigten eine Tumorantwort (1 Komplettremission, 2 partielle Remissionen, 3 geringe Remissionen). Das mediane Überleben aller Patienten lag über zwölf Monaten. 
Zurzeit werden Antisense-Therapien bei einem breiten Spektrum von Erkrankungen eingesetzt, insbesondere im onkologischen Bereich. Dabei werden neue Modifikationen von Antisense-Molekülen entwickelt, die höhere Halbwertszeiten und geringere Toxizitäten aufweisen. Antisense-Moleküle werden nicht mehr nur für ein bestimmtes Zielgen eingesetzt, sondern z. B. auch, um alternatives splicing zu reduzieren, welches häufig mit einer Tumorprogression einhergeht. Auch die Anwendung von RNAi (RNA interference) ist eine viel versprechende Anwendungsmöglichkeit. Bei diesem Verfahren soll es nach RNAi-Anwendung zur Bildung von Doppelstrang-RNA des Zielgens kommen und somit zur nachfolgenden Degradation der mRNA und Reduktion der Translation. Neben inhibitorischen Effekten wurde auch gezeigt, dass Antisense-Oligonukleotide immunstimulatorische Effekte durch z.B. CpG-Motive induzierten. Diese CpGOligonukleotide können zur Optimierung von immunstimulatorischen Therapien in der Krebsbehandlung genutzt werden. Je mehr Erfahrungen sich bei der Anwendung von Antisense-Oligonukleotiden ergeben, umso mehr neue Fragen werden aufgeworfen. Die derzeitigen Beobachtungen zeigen jedoch, dass Antisense-Nukleotide ein Potenzial haben, therapeutische Effekte zu bewirken, insbesondere in Kombination mit anderen Therapien wie z.B. der Chemotherapie.
${ }^{1}$ Fredholm BB, Abbracchio MP, Burnstock G, Daly JW, Harden TK, Jackobson KA, Leff P, Williams B. Nomenclature and classification of purinoceptors. Pharmacol Rev 1994; 46: 143 - 156

2 Simon MI, Strathmann MP, Gautam N. Diversity of G proteins in signal transduction. Sience 1991; 252: $802-808$

${ }^{3}$ Birnbaumer L. Receptor-to-effector signaling through G proteins: roles for beta gamma dimers as well as alpha subunits. Cell 1992; 71: $1069-1072$

${ }^{4}$ Offermanns S, Schultz G. Complex information processing by the transmembrane signaling system involving $G$ proteins. NauynSchmiedeberg's Arch Pharmacol1994; 350: 329-338

${ }^{5}$ Birnbaumer L, Birnbaumer M. Signal transduction by G proteins: 1994 edition. J Receptor and Signal Transduction Res 1995; 15: 213 -252

${ }^{6}$ Neer EJ. Heterotrimeric $G$ proteins: organizers of transmembrane signals. Cell 1995; 80: 249-257

${ }^{7}$ Milligan G. Signal sorting by G-protein-linked receptors. Adv Pharmacol1995; 32: $1-28$

${ }^{8}$ Nürnberg B, Gudermann T, Schultz GJ. Receptors and G proteins as primary components of transmembrane signal transduction. Part 2. G proteins: structure and function. Mol Med 1995; 73: 123 - 132

${ }^{9}$ Kalkbrenner F, Dippel E, Wittig B, Schultz G. Specifity of interaction between receptor and $\mathrm{G}$ protein: use of antisense techniques to relate G-protein subunits to function. Biochem Biophys 1996; 1314: 125 139

${ }^{10}$ Ramkumar V, Stiles GL, Beaven MA, Ali H. The A3 adenosine receptor is the unique adenosine receptor which faciliates release of allergic mediators in mast cells. Biol Chem 1993; 268: 16887-16890

${ }^{11}$ Dippel E, Kalkbrenner F, Wittig B, Schultz G. A heterotrimeric G protein complex couples the muscarinic $\mathrm{m} 1$ receptor to phospholipase C-beta. Proc Natl Acad Sci (USA) 1996; 93: 1391-1396

${ }^{12}$ Crooke ST. Antisense strategies. Curr Mol Med 2004; 4: 465- 487

${ }^{13}$ Büchele T. Proapoptotische Therapie mit Oblimersen (bcl-2-Antisense-Oligonukleotid) - Übersicht über präklinische und klinische Daten. Onkologie 2003; 26: 60-69

${ }^{14}$ Jansen B, Wacheck V, Heere-Ress E, Schlagbauer-Wadl H, Hoeller C, Lucas T, Hoermann M, Hollenstein U, Wolff K, Pehamberger H. Chemosensitisation of malignant melanoma by BCL2 antisense therapy. Lancet $2000 ; 356$ : $1728-1733$

\section{Personalia}

Herrn Prof. Dr. Dr. h.c. Constantin Orfanos (Berlin) wurde nach über 25-jähriger Tätigkeit als Direktor der Klinik und Poliklink für Dermatologie am Klinikum Benjamin Franklin der FUB am 1.10. 2004 emeritiert. Bei diesem Anlass wurde ihm vom medizinischen Vizepräsidenten der FU, Herrn Prof. Tauber, und vom Dekan der Charité - Universitätsmedizin Berlin, Herrn Prof. Paul für seine langjährigen Verdienste um den Fachbereich Humanmedizin der Freien Universität Berlin die „Benjamin Franklin Medaille“ in Silber verliehen. 